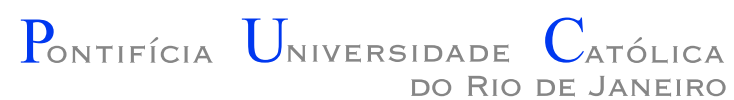

Felipe Freixo Pina

\title{
Aplicações de DHT em sistemas de computação distribuída
}

\author{
Dissertação de Mestrado
}

\begin{abstract}
Dissertação apresentada como requisito parcial para obtenção do grau de Mestre pelo Programa de Pós-graduação em Informática do Departamento de Informática da PUC-Rio
\end{abstract}

Orientador: Prof. Noemi de La Rocque Rodriguez 
Felipe Freixo Pina

\title{
Aplicações de DHT em sistemas de computação distribuída
}

Dissertação apresentada como requisito parcial para obtenção do grau de Mestre pelo Programa de Pós-graduação em Informática do Departamento de Informática do Centro Técnico Científico da PUC-Rio. Aprovada pela comissão examinadora abaixo assinada.

\author{
Prof. Noemi de La Rocque Rodriguez \\ Orientador \\ Departamento de Informática - PUC-Rio
}

Prof. Markus Endler

Departamento de Informática - PUC-Rio

Prof. Bruno Oliveira Silvestre

Instituto de Informática - Universidade Federal de Goiás

Prof. José Eugenio Leal

Coordenador do Centro Técnico Científico - PUC-Rio

Rio de Janeiro, 29 de Agosto de 2011 
Todos os direitos reservados. Proibida a reprodução total ou parcial do trabalho sem autorização da universidade, do autor e do orientador.

\section{Felipe Freixo Pina}

Graduou-se em Engenharia de Computação pela Pontifícia Universidade Católica do Rio de Janeiro em 2002.

Ficha Catalográfica

Pina, Felipe Freixo

Aplicações de DHT em sistemas de computação distribuída / Felipe Freixo Pina; orientador: Noemi de La Rocque Rodriguez. - Rio de Janeiro : PUC-Rio, Departamento de Informática, 2011.

v., 63 f: il. ; $29,7 \mathrm{~cm}$

1. Dissertação (Mestrado em Informática) - Pontifícia Universidade Católica do Rio de Janeiro, Departamento de Informática.

Inclui referências bibliográficas.

1. Informática - Tese. 2. Sistemas Distribuídos. 3. P2P. 4. DHT. 5. Lua. 6. Grupos. I. Rodriguez, Noemi de La Rocque. II. Pontifícia Universidade Católica do Rio de Janeiro. Departamento de Informática. III. Título. 


\section{Agradecimentos}

À minha família, pelo incentivo.

À Noemi, pela ajuda e paciência.

E à Renata, pela compreensão e apoio nos momento difíceis. 


\section{Resumo}

Pina, Felipe Freixo; Rodriguez, Noemi de La Rocque. Aplicações de DHT em sistemas de computação distribuída. Rio de Janeiro, 2011. 63p. Dissertação de Mestrado — Departamento de Informática, Pontifícia Universidade Católica do Rio de Janeiro.

Arquiteturas P2P destacam-se pela descentralização e pelo incentivo a cooperação entre nós. Essas características permitem que sistemas baseados nesta arquitetura sejam tolerantes a falhas e que os recursos sejam distribuídos entre os nós (via replicação). A utilização da técnica de DHT na criação de redes P2P permite que os sistemas sejam escaláveis. Ao contrário do uso mais comum em sistemas de distribuição de conteúdo, este trabalho investiga aplicações da técnica de DHT em sistemas de computação distribuída, onde o recurso compartilhado é a capacidade de processamento de cada nó. Quatro protocolos de roteamento de mensagens foram analisados para identificar os mais adequados aos sistemas de computação distribuída e aplicou-se o conceito de grupo de nós com o objetivo de aumentar a tolerância a falhas e distribuir tarefas entre os nós da rede.

\section{Palavras-chave}

Sistemas Distribuídos; P2P; DHT; Lua; Grupos; 


\section{Abstract}

Pina, Felipe Freixo; Rodriguez, Noemi de La Rocque (Advisor). Utilization of DHT in distributed computing systems. Rio de Janeiro, 2011. 63p. MSc. Dissertation — Departamento de Informática, Pontifícia Universidade Católica do Rio de Janeiro.

P2P architectures are recognized for decentralization and incentive for the cooperation among nodes. These characteristics allow for fault tolerance and resource distribution among the nodes (by replication) to systems based on the P2P architecture. Systems based in P2P networks built using the DHT technique are scalable. Since this architecture is commonly used in content distribution systems, in this work we investigate the utilization of the DHT technique in distributed computing systems, where the shared resources are the node's computational power. Four routing protocols were analyzed to identify the most appropriated for use in distributed computing systems and applied the group concept to archive fault tolerance and resource distribution among nodes.

\section{Keywords}

Distributed Systems; P2P; DHT; Lua; Group membership; 


\section{Sumário}

1 Introdução $\quad 11$

$\begin{array}{ll}1.1 \text { Motivação } & 12\end{array}$

$\begin{array}{lll}1.2 & \text { Objetivos e contribuições } & 13\end{array}$

$\begin{array}{lll}1.3 & \text { Estrutura da Dissertação } & 14\end{array}$

2 Arquiteturas P2P $\quad 15$

$\begin{array}{lll}2.1 & \text { Redes não-estruturadas } & 16\end{array}$

$\begin{array}{lll}2.2 & \text { Redes estruturadas } & 17\end{array}$

$\begin{array}{ll}2.3 & \text { Distributed Hash Table } \\ & 17\end{array}$

2.4 Avaliação dos protocolos 27

3 Sistemas distribuídos em arquiteturas P2P 33

3.1 Cassandra 33

$\begin{array}{lll}3.2 & \text { Squirrel } & 33\end{array}$

3.3 Computação distribuída 34

4 Aplicações da técnica de DHT em sistemas de computação distribuída 39

4.1 Implementação $\quad 39$

4.2 Camada DHT 47

5 Avaliação experimental $\quad 49$

5.1 Avaliação quantitativa 49

5.2 Avaliação qualitativa 54

6 Conclusão $\quad 58$

6.1 Trabalhos Futuros $\quad 59$

$\begin{array}{lc}\text { Referências Bibliográficas } & 60\end{array}$ 


\section{Lista de figuras}

2.1 Exemplo de um círculo de identificação

2.2 Estado de um nó com nodeld $=10233102$ e $b=2$. Os números estão na base 4. A primeira linha da tabela de roteamento representa o nível zero.

2.3 Ilustração do roteamento de mensagens na rede Pastry.

2.4 Crescimento do tamanho médio das rotas em função da quantidade de nós

3.1 Exemplo de arquitetura de uma aplicação ALua 37

3.2 Modelo do ALua 38

4.1 Diagrama dos módulos do ALua 40

4.2 Exemplo de rede formada pela técnica de DHT 41

4.3 Exemplo multicast 42

5.1 Tempo médio de comunicação do protocolo Chord no cluster 51

5.2 Distribuição dos tamanhos das rotas do protocolo Chord no cluster 51

5.3 Tempo médio de comunicação do protocolo Pastry no cluster 52

5.4 Distribuição dos tamanhos das rotas do protocolo Pastry no cluster 52

5.5 Tempo médio de comunicação no PlanetLab para $\mathrm{N}=75 \quad 53$

5.6 Distribuição dos tamanhos das rotas no PlanetLab para $\mathrm{N}=75 \quad 54$

5.7 Exemplo de partição do mapa do jogo 56 


\section{Lista de tabelas}

2.1 Informações presentes na tabela de roteamento 19

2.2 Comparação da escalabilidade 28

2.3 Tolerância a falhas 30

2.4 Avaliação do desempenho 31

5.1 Tempos médios de comunicação no cluster 50

5.2 Tamanhos médios das rotas no cluster 50

5.3 Tempos e tamanhos de rotas médios no PlanetLab para $\mathrm{N}=75 \quad 53$

5.4 Tempos médios da multiplicação de matrizes $\quad 55$ 
Without Education, we are in horrible and deadly danger of taking educated people seriously.

G.K. Chesterton, the Illustrated London News. 